MATEC Web of Conferences 23, 01002 (2015)

DOI: $10.1051 /$ matec conf/ 20152301002

(C) Owned by the authors, published by EDP Sciences, 2015

\title{
Heating of the liquid film flowing under gravity and gas flow
}

\author{
Sergey P. Aktershev ${ }^{1,2 a}$, Maria V. Bartashevich ${ }^{1}$ \\ ${ }^{1}$ Kutateladze Institute of Thermophysics, Novosibirsk, Russia \\ ${ }^{2}$ Novosibirsk State University, Novosibirsk, Russia
}

\begin{abstract}
The lliquid films are applied in the various equipments intended for an intensification of heat and mass transfer processes. Heat transfer can also be intensifying by the gas flow above the liquid surface; these processes take a place at two-phase flows in mini- and microchannels. This study deals with the heat transfer in a locally heated liquid film of constant thickness $h$. The film is supposed to flow along the heated plate having constant temperature $T_{W}$ under the action of gravity and a gas flow. Influence of a gas flow on a velocity profile in the liquid is considered through shear stress on an interface. Coefficient of heat exchange and shear stress on a film surface suppose be set. Equation of the energy for a liquid film is solved by Galerkin technique which is special case of the weighed residuals method (WRM). Applying of Galerkin technique to this problem allows calculating the temperature in a liquid analytically for arbitrary a temperature profile in initial cross-section of a film. For initial temperature profile, gained from the analytical solution the temperature field is calculated. Results of these calculations are compared to results of numerical solution of the energy equation by finite-difference method. This comparison has shown the good agreement both for a falling film, and for cocurrent or countercurrent gas flow.
\end{abstract}

\section{Problem statement}

Let us consider a stationary laminar flow of an inclined liquid film with constant thickness $h$ along a hot wall with temperature $T_{W}$. The liquid contacts to the moving gas, having the temperature $T_{g}$. Heat exchange between a liquid and gas is described by the heat exchange coefficient $\alpha$. Density $\rho$, kinematic viscosity $v$, heat conductivity $\lambda$, thermal diffusivity $a$ of the liquid are supposed to be constants. Let's introduce Cartesian coordinate system $O x y$ with $O x$ axis in the direction of gravity, and $O y$ axis normal to the plate (the edge of a heater matches $x=0$ ). Temperature field in a liquid is described by the equation of energy which in boundary layer approach $\left(\partial^{2} / \partial x^{2}<<\partial^{2} / \partial y^{2}\right)$ looks like

$$
u(y) \frac{\partial T}{\partial x}=a \frac{\partial^{2} T}{\partial y^{2}},
$$

where $u=(g \sin \theta / 2 v)\left(2 y h-y^{2}\right)+y \tau / \rho v$ is velocity of a liquid in a film, $\tau$ is a shear stress at the interface due to the gas flow, $\theta$ is an inclination angle to the horizon, $g$ is acceleration of gravity. We choose $l=3 \operatorname{Pr} \operatorname{Re}_{m} h / 2$ as the scale on $O x$ axis and $\Delta T=T_{W}-T_{g}$ as the temperature scale.

\footnotetext{
a Corresponding author: sergey-aktershev@mail.ru
}

This is an Open Access article distributed under the terms of the Creative Commons Attribution License 4.0, which permits unrestricted use, distribution, and reproduction in any medium, provided the original work is properly cited. 


\section{MATEC Web of Conferences}

Here $\operatorname{Re}_{m}=g h^{3} \sin \theta / 3 v^{2}$ is Reynolds number of the falling film, $\operatorname{Pr}=v / a$ is Prandtl number. We introduce dimensionless variables $x / l, y / h,\left(T-T_{g}\right) / \Delta T$, keeping the same notations. In dimensionless variables the equation (1) looks like

$$
\left((2+r) y-y^{2}\right) \frac{\partial T}{\partial x}=\frac{\partial^{2} T}{\partial y^{2}} .
$$

Here $r=2 \tau /(\rho g h \sin \theta)$ is a dimensionless shear stress. Boundary conditions for equation (2) at the heated wall and at the film surface look as follows

$$
\left.T\right|_{y=0}=1,\left.\left(\frac{\partial T}{\partial y}+B i \cdot T\right)\right|_{y=1}=0 .
$$

Here $B=\alpha h / \lambda$ is a Biot number. The conditions (3) should be provided by a profile of temperature of a liquid on an inlet (at $x=0)$ :

$$
\left.T\right|_{x=0}=F(y) .
$$

Note, that the problem similar to (2)-(4) for laminar liquid flow in a tube in a region of thermal stabilization has been solved by Petukhov [1] by expansion the temperature into the series of degrees of radial coordinate.

\section{Applying of Galerkin technique to the film flow heating}

We solve this problem by Galerkin technique which is special case of the weighed residuals method (WRM) [2]. We search for the temperature field in the form

$$
T(x, y)=T_{s t}-\sum_{k=1}^{\infty} a_{k}(x) \psi_{k}(y) .
$$

Here $T_{s t}=1-y \cdot B i /(1+B i)$ is a temperature profile in a film which will be established far from heater edge, and test functions $\psi_{k}(y) \equiv \sin \omega_{k} y, k=1,2,3 \ldots$ should satisfy to boundary conditions

$$
\psi(0)=0,\left.(d \psi / d y+B i \cdot \psi)\right|_{y=1}=0 .
$$

It follows from (6) that all frequencies $\omega_{k}$ must be roots of the transcendental equation

$$
\omega \cdot \cos \omega+B i \cdot \sin \omega=0 \text {. }
$$

The system of test functions $\psi_{k}(y)$ defined in the interval $0 \leq y \leq 1$ can serve as orthogonal basis (that is $\int_{0}^{1} \psi_{k} \psi_{m} d y$ at $\left.k \neq m\right)$. Any function $\Phi(y)$ can be presented in the form of an expansion in a series on basis functions $\psi_{k}(y)$. It is easy to see, that the temperature defined by formula (6) satisfies to boundary conditions (3). Substituting (5) in the equation (2), we receive a residual

$$
R(x, y)=\sum_{k=1}^{\infty} R_{k}(x, y) \equiv \sum_{k=1}^{\infty}\left(\left((2+r) y-y^{2}\right) \frac{d a_{k}}{d x}+\omega_{k}^{2} a_{k}\right) \psi_{k} .
$$

Equating to null a projection of any residual component $R_{k}(x, y)$ on the element of basis $\psi_{k}(y)$, we obtain the equation for "amplitude" $a_{k}(x)$.

$$
\frac{d a_{k}}{d x} \int_{0}^{1}\left((2+r) y-y^{2}\right) \psi_{k}^{2} d y+\omega_{k}^{2} a_{k} \int_{0}^{1} \psi_{k}^{2} d y=0
$$


The solution of this equation looks like

$$
\left.a_{k}(x)=a_{k}(0) \exp \left(-\lambda_{k} x\right), \text { where } \lambda_{k}=\omega_{k}^{2} \int_{0}^{1} \psi_{k}^{2} d y / \int_{0}^{1}\left((2+r) y-y^{2}\right)\right) \psi_{k}^{2} d y>0 .
$$

The values $a_{k}(0)$ should be calculated with help of conditions (4) on an inlet. Substituting (5) in (4), we obtain

$$
\sum_{k=1}^{\infty} a_{k}(0) \psi_{k}(y)=T_{s t}(y)-F(y) .
$$

Thus, $a_{k}(0)$ are expansion coefficients of function $T_{s t}-F$ in basis ${ }_{k}(y)$. Taking into account orthogonality of basic functions, all $a_{k}(0)$ are calculated by the formula

$$
a_{k}(0)=\int_{0}^{1}(1-y B i /(1+B i)-F(y)) \psi_{k} d y / \int_{0}^{1} \psi_{k}^{2} d y .
$$

Thus, relations (5), (7), (8) represent the solution of a problem by Galerkin method for an arbitrary profile of temperature on an inlet. The inlet temperature profile $F(y)$ we take through the self-similar solution which is true for the entrance region $(x<<1)$. This self-similar solution looks as [3]

$$
T_{a}(\xi)=1-\int_{0}^{\xi} \exp \left(-z^{3} / 9\right) d z / \int_{0}^{\infty} \exp \left(-z^{3} / 9\right) d z
$$

Here $\xi=y((2+r) / x)^{1 / 3}$ is self-similar variable. Here $x$ is a distance, which is necessary for growing up of the heated layer to the film surface.

\section{Results of the calculations}

In figures 1-2 the results of calculations by WRM compared with numerical solutions of the equation (2) by finite difference method are presented for vertical film flow. Opened circles show calculation by WRM and solid lines show calculation by finite difference method. In figure 1 the surface temperature of the falling film in dependence on distance is presented. The curves 1-2 correspond to different values of Biot number. Figure 2 show the surface temperature in depends on distance for liquid film flowing under gravity and countercurrent gas flow. The curves 1-3 correspond to the different values of the dimensionless shear-stress $r$ at fixed Biot number $B i=0.5$.

The distance on which the temperature profile becomes close to $T_{s t}(y)$, decreases with growth of Biot number (see Fig. 1). This distance essentially depends on a shear stress $\tau$. For example, in case of countercurrent gas flow $(r<0)$, the distance of development of temperature profile decrease essentially with growth of $|r|$ (see Fig. 2). 


\section{MATEC Web of Conferences}

Figure 1. Surface temperature versus distance for different values of Biot number. $1-B i=0.1,2-B i=0.5$.

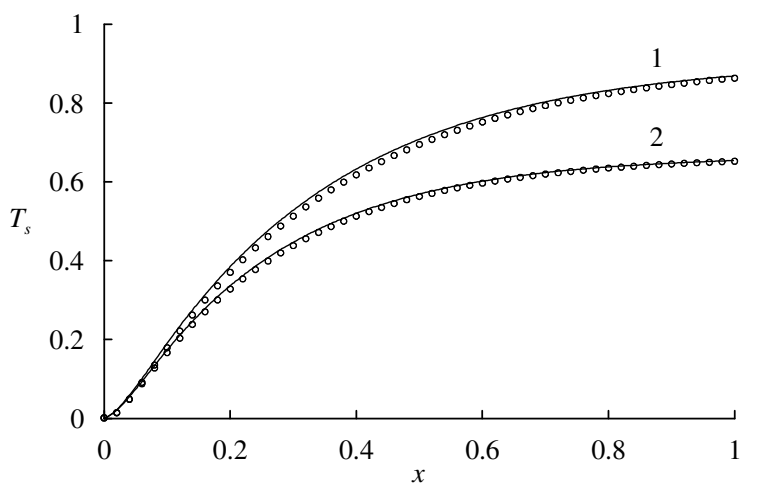

Figure 2. Surface temperature versus distance for various values of $r .1-r=0,2-r=-0.5,3-r=-1$.

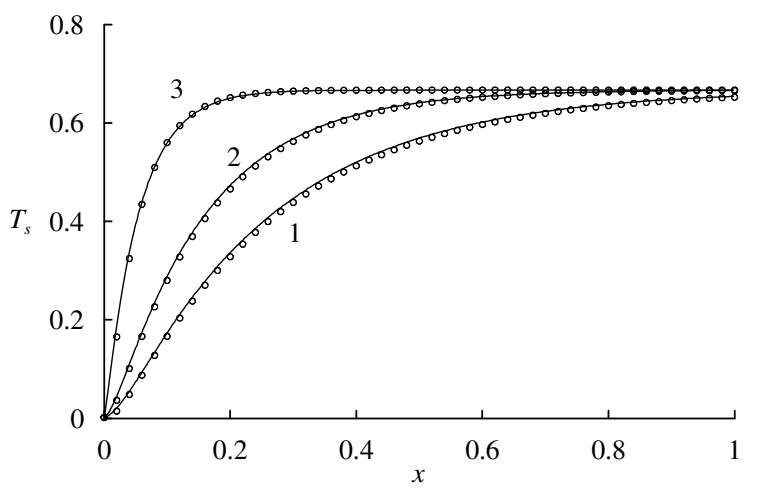

\section{Conclusion}

The new analytical method of calculation of heat transfer based on applying of weighed residuals method to problem of heating of liquid films is proposed. Results of calculations of temperature in the film flowing under the action of gravity and a gas flow well agree with numerical solutions of energy equation by finite difference method. The good agreement takes place both for a falling film, and for case of co-current or countercurrent gas flow.

\section{Acknowledgment}

This work was supported by the grant of RSCF № 15-19-10025.

\section{References}

1. B. S. Petukhov, Heat Transfer and Hydraulic Resistance of Laminar Liquid Flow in Tubes (Energy, Moscow, 1967)

2. C. A. J. Fletcher, Computational Techniques for Fluid Dynamics, Vol. 1 and 2 (Springer-Verlag, 1987)

3. V. E. Nakoryakov and A. V. Gorin, Teplomassoperenos $v$ dvukhfaznykh sistemakh (Heat and Mass Transfer in Two-Phase Systems) (Novosibirsk: Institute of Thermophysics SB RAS, 1994) 